\title{
Experimental Optimization of Self-Priming Pump Based on the Full Factor Test
}

\author{
Hao CHANG***, Shiming HONG***, Dehui YU****, Guangjie PENG*****, \\ Daoxing YE******, Jianrui LIU***, Shouqi YUAN*** \\ *Research Center of Fluid Machinery Engineering and Technology, Jiangsu University, Zhenjiang 212013, China \\ **Key Laboratory of Fluid Machinery and Engineering (Xihua University), Sichuan Province, 610039, China \\ ***Research Center of Fluid Machinery Engineering and Technology, Jiangsu University, Zhenjiang 212013, China \\ ****Chongqing Pump Industry Co. Ltd., Chongqing, 400033, China \\ *****Research Center of Fluid Machinery Engineering and Technology, Jiangsu University, Zhenjiang 212013, China, \\ E-mail:pgj@ujs.edu.cn (Corresponding Author) \\ ******Key Laboratory of Fluid Machinery and Engineering (Xihua University), Sichuan Province, 610039, China, \\ E-mail: dxingye@163.com (Corresponding Author) \\ crossref http://dx.doi.org/10.5755/j02.mech.29931
}

\section{Introduction}

Self-priming pump [1] as the core equipment for flood fighting and emergency rescue, play an important role in flood prevention and other fields. Plenty of scholars pay a lot of attention on the performance improvement of selfpriming pump. Wu et al. [2] proposed five different blade profiles impellers to study the energy performance of selfpriming pump. Zhou et al. [3] investigated the influence of the reflux hole on the transient startup performance of the self-priming centrifugal pump. Yao et al. [4] employed the Fluent to analyse the gas-liquid two-phase unsteady characteristic of vertical self-priming pump, which could provide the reference to the design method of high-head vertical self-priming pump. Zhang et al. [5] study the transient hydraulic performance of a self-priming pump with different start-up condition by experiment. Wang et al. [6] analyzed self-priming process of multistage self-priming centrifugal pump by the numerical simulation, internal flow field of air water two-phase was obtained based on ANSYS CFX, and the analysis results were verified by self-priming test. However, most of the researcher focus on the total structure optimization of self-priming pump to improve performance, only few researchers pay attention on the experimental optimization of self-priming pump by modifying the geometry parameters of self-priming nozzle. Herein, in this paper, the full factor test method is employed to explore the main and interaction effect of the nozzle geometric parameters on the self-priming performance, and the order of response to selfpriming time is obtained. Meanwhile, a novel self-priming performance model was established, the self-priming time of different geometric parameters self-priming nozzles can be effectively predicted, the test analysis cycle and production costs were greatly reduced. Finally, the self-priming test under different self-priming heights with optimal selfpriming nozzle was conduct, the variation regulation between self-priming time and self-priming height was obtained. Therefore, this research could effectively provide the design reference of self-priming nozzles optimization.

\section{Experiential analysis method}

According to the previous investigation of the selfpriming nozzle [7], it found that the geometry of the self- priming nozzle plays an essential part on the startup performance. Meanwhile, the relationship between the self-priming time and nozzle geometry parameters based on the gray correlations analyze was obtained. However, research on the optimization of self-priming nozzles is relatively lacking, which cannot guarantee whether the effects of geometric parameters of the self-priming nozzle on the self-priming time are independent of each other, and whether there is an interaction between any two of the three parameters. Therefore, in order to eliminate the possible deviations in the analysis process, the effect of second order interaction between the parameters investigated in this paper.

The full factor test method can load each level of each parameter in the analysis process, and more comprehensive test schemes and more sufficient statistical analysis results are obtained. This method can not only analyze the main effects of the parameters, but also effectively evaluate the interaction between the parameters. Therefore, the full factor test method is employed in this paper to explore the main and interaction effect of the nozzle geometric parameters on the self-priming performance, which can further verify the analysis results of the gray correlation method.

\section{Selection of experiment schemes}

The geometric parameters of the first-stage nozzle diffuser outlet inner diameter $A$, the connecting sleeve length $B$, and the second throat pipe inner diameter of second-stage nozzle $C$ are selected as the research variables, as shown in Fig. 1. What' more, there are three levels under each group of variables, and 27 test schemes are generated based on the full factor test, the independent repeated selfpriming test is conducted twice for each group of schemes. The test schemes and corresponding test results as shown in Table 1.

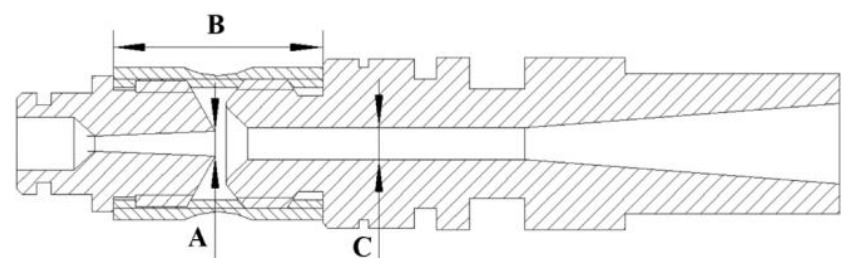

Fig. 1 Structure diagram of self-priming nozzle 


\section{Full factor test analysis}

\subsection{Analysis of main effect}

Fig. 2 shows the main effect diagrams of three selfpriming nozzle geometric parameters on the self-priming time. As shown in Fig. 2, a, it founded that with the continuous increase of the outlet inner diameter $A$, the average self-priming time gradually decreases, and the rate of decrease is significantly higher than main effect of other schemes, namely, the response of outlet inner diameter $A$ on the self-priming time is more significant. At the same time, according to the investigation of main effect diagram of connecting sleeve length $B$ on the average self-priming time, it can be seen that with the continuous increase of connecting sleeve length $B$, the self-priming time also presents a decreasing trend, but the decrease rate is obviously smaller than outlet inner diameter $A$. Furthermore, the relationship between the main effect of throat pipe inner diameter $C$ and average self-priming time is shown in Fig. 2, c, the average self-priming time decreases first and then increases as the throat pipe inner diameter $C$ increases, and reaches the minimum when $C=5.5 \mathrm{~mm}$. In addition, the variation rate of average self-priming time with nozzle geometric parameters is shown as follows: outlet inner diameter $A>$ throat inner diameter $C>$ the connecting sleeve length $B$. Hence, the order of the significant degree of response on the self-priming time is obtained: outlet inner diameter $A>$ throat inner diameter $C>$ connecting sleeve length $B$.

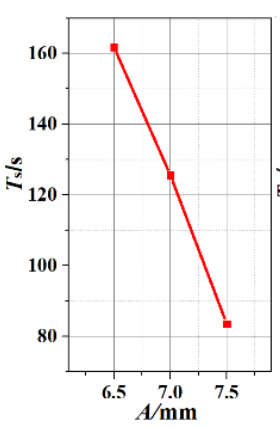

a

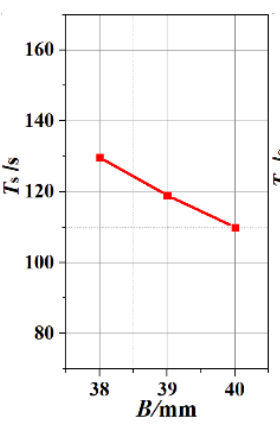

b

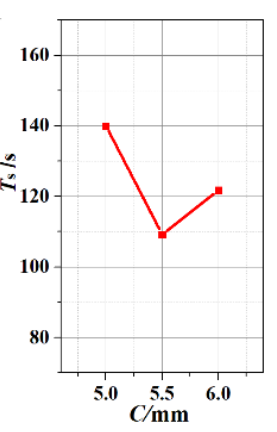

Fig. 2 Main effect diagram of each parameter: a) Main effect of $A$; b) Main effect of $B$; c) Main effect of $C$

Selection of experiment schemes

\begin{tabular}{|c|c|c|c|}
\hline Scheme & The first self-priming time, $\mathrm{s}$ & The second self-priming time, $\mathrm{s}$ & The average self-priming time, $\mathrm{s}$ \\
\hline$A_{1} B_{1} C_{1}$ & 168 & 164 & 166 \\
\hline$A_{1} B_{2} C_{1}$ & 175 & 184 & 179.5 \\
\hline$A_{1} B_{3} C_{1}$ & 169 & 168 & 168.5 \\
\hline$A_{2} B_{1} C_{1}$ & 142 & 136 & 139 \\
\hline$A_{2} B_{2} C_{1}$ & 140 & 134 & 137 \\
\hline$A_{2} B_{3} C_{1}$ & 113 & 125 & 119 \\
\hline$A_{3} B_{1} C_{1}$ & 101 & 97 & 99 \\
\hline$A_{3} B_{2} C_{1}$ & 143 & 117 & 130 \\
\hline$A_{3} B_{3} C_{1}$ & 124 & 120 & 122 \\
\hline$A_{1} B_{1} C_{2}$ & 124 & 123 & 123.5 \\
\hline$A_{1} B_{2} C_{2}$ & 122 & 118 & 120 \\
\hline$A_{1} B_{3} C_{2}$ & 208 & 256 & 232 \\
\hline$A_{2} B_{1} C_{2}$ & 135 & 134 & 134.5 \\
\hline$A_{2} B_{2} C_{2}$ & 91 & 90 & 90.5 \\
\hline$A_{2} B_{3} C_{2}$ & 91 & 88 & 89.5 \\
\hline$A_{3} B_{1} C_{2}$ & 69 & 63 & 66 \\
\hline$A_{3} B_{2} C_{2}$ & 65 & 64 & 64.5 \\
\hline$A_{3} B_{3} C_{2}$ & 63 & 62 & 62.5 \\
\hline$A_{1} B_{1} C_{3}$ & 135 & 134 & 134.5 \\
\hline$A_{1} B_{2} C_{3}$ & 142 & 143 & 142.5 \\
\hline$A_{1} B_{3} C_{3}$ & 206 & 174 & 190 \\
\hline$A_{2} B_{1} C_{3}$ & 146 & 149 & 147.5 \\
\hline$A_{2} B_{2} C_{3}$ & 90 & 92 & 91 \\
\hline$A_{2} B_{3} C_{3}$ & 93 & 92 & 92.5 \\
\hline$A_{3} B_{1} C_{3}$ & 67 & 68 & 67.5 \\
\hline$A_{3} B_{2} C_{3}$ & 68 & 66 & 67 \\
\hline$A_{3} B_{3} C_{3}$ & 71 & 76 & 73.5 \\
\hline
\end{tabular}

\subsection{Interaction effect analysis}

Fig. 3 shows the interaction diagram of two parameters, which means that one parameter kept constant, and the response of the second-order interaction formed by the other two parameters on the average self-priming time. In general, the parallel degree between the curves is applied to evaluate the response of the interaction between the nozzle geometry parameters and average self-priming time. As shown in Fig. 3, a, it presents that when the throat pipe inner diameter
$C$ remains constant, the influence of interaction between the outlet inner diameter $A$ and connecting sleeve length $B$ on the average self-priming time. It found that significant interactions were generated within three curves, that is, the interaction between outlet inner diameter $A$ and connecting sleeve length $B$ has a significant response on the average self-priming time. However, according to Fig. 3, b, and Fig. 3, c, the interaction between outlet inner diameter $A$ and the throat pipe inner diameter $C$, the connecting sleeve length $B$ and the throat pipe inner diameter $C$ are all parallel 
curves, indicating that the interaction between the two pa- priming time. rameters does not respond significantly to the average self-

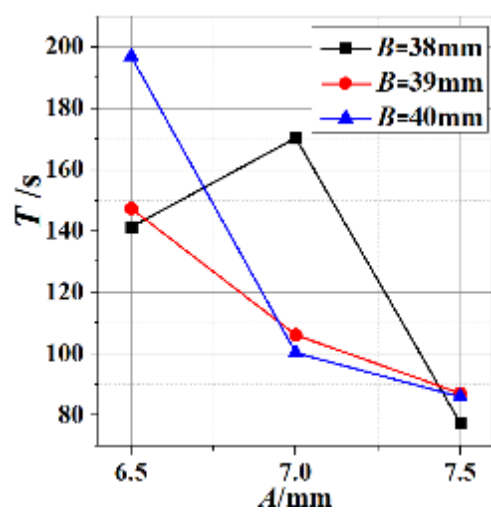

a

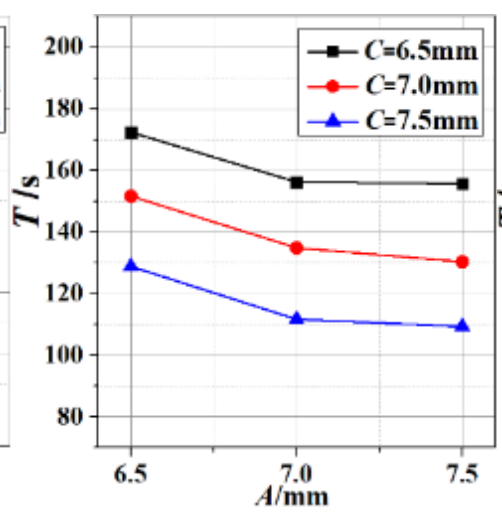

$\mathrm{b}$

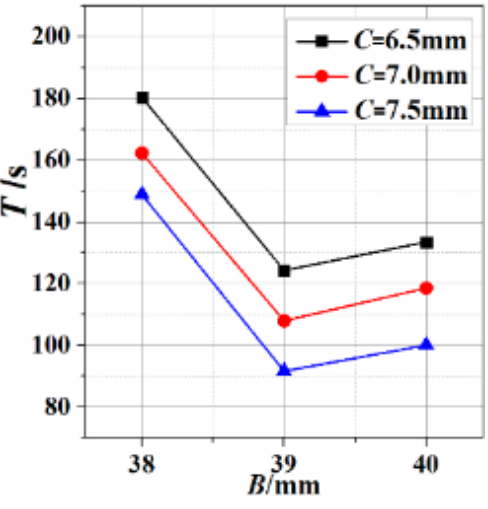

C

Fig. 3 Interaction diagram of parameters: a) $A$ and $B$; b) $A$ and $C$; c) $B$ and $C$

4.3. Self-priming performance response model

In order to investigate the influence of the geometric parameters of the first-stage nozzle diffuser outlet inner diameter $A$, the connecting sleeve length $B$, and the second throat pipe inner diameter of second-stage nozzle $C$, and second-order interaction on the self-priming time, all factors were considered in analysis. Meanwhile, the response model between the above six parameters and self-priming time established by Minitab software is:

$$
\begin{aligned}
& T_{s 1}=-6944+1014 A+168 B+189 C- \\
& -23.5 A * B-32 A * C+0.2 B * C .
\end{aligned}
$$

Error analysis of the model

Table 2

\begin{tabular}{|c|c|c|c|c|c|c|c|}
\hline$A, \mathrm{~mm}$ & $B, \mathrm{~mm}$ & $C, \mathrm{~mm}$ & $T_{\mathrm{s}, \mathrm{s}}$ & $T_{\mathrm{s} 1}, \mathrm{~s}$ & Original model error, $\%$ & $T_{\mathrm{s} 2}, \mathrm{~s}$ & Simplified model error, $\%$ \\
\hline 7.5 & 40 & 5.0 & 122 & 169.5 & -2.0649 & 170.93 & -2.96988 \\
\hline 7.5 & 40 & 6.0 & 73.5 & 185.75 & -3.36474 & 174.115 & 3.092784 \\
\hline 6.5 & 39 & 5.0 & 179.5 & 202 & -16.5842 & 177.3 & -5.22255 \\
\hline 7.0 & 40 & 5.5 & 89.5 & 150 & -7.33333 & 131.54 & 5.366906 \\
\hline 7.0 & 38 & 5.0 & 139 & 154.5 & -11.3269 & 134.97 & 1.481752 \\
\hline 7.5 & 38 & 5.0 & 99 & 159 & -25.1572 & 138.4 & -16.3025 \\
\hline 6.5 & 38 & 5.5 & 123.5 & 130.5 & -24.1379 & 92.15 & 6.919192 \\
\hline 6.5 & 40 & 5.0 & 168.5 & 123.25 & 5.476673 & 95.825 & 26.28846 \\
\hline 7.5 & 40 & 5.5 & 62.5 & 116 & 5.172414 & 99.5 & 18.44262 \\
\hline 7.5 & 38 & 6.0 & 67.5 & 163.8 & -24.6032 & 156.83 & -26.9879 \\
\hline 6.5 & 39 & 6.0 & 142.5 & 180.15 & -33.3888 & 160.015 & -25.007 \\
\hline 6.5 & 39 & 5.5 & 120 & 196.5 & 18.06616 & 163.2 & 29.65517 \\
\hline 7.0 & 39 & 5.5 & 90.5 & 136.3 & -1.32062 & 117.44 & 12.68401 \\
\hline 7.0 & 40 & 6.0 & 92.5 & 140.9 & -35.77 & 120.87 & -25.1262 \\
\hline 7.0 & 39 & 6.0 & 91 & 145.5 & -38.488 & 124.3 & -27.9968 \\
\hline 6.5 & 40 & 6.0 & 190 & 108.8 & -39.3382 & 78.05 & -18.2576 \\
\hline 6.5 & 38 & 6.0 & 134.5 & 101.65 & -36.547 & 81.725 & -26.7054 \\
\hline 6.5 & 38 & 5.0 & 166 & 94.5 & -33.8624 & 85.4 & -26.815 \\
\hline 7.5 & 39 & 5.5 & 64.5 & 158.1 & -14.9273 & 142.73 & -6.11896 \\
\hline 7.0 & 39 & 5.0 & 137 & 174.55 & -18.3615 & 145.915 & -2.39649 \\
\hline 7.0 & 38 & 6.0 & 147.5 & 191 & -0.52356 & 149.1 & 21.52632 \\
\hline 7.5 & 39 & 6.0 & 67 & 122.6 & 20.30995 & 103.34 & 29.93898 \\
\hline 7.0 & 40 & 5.0 & 119 & 127.3 & -28.5153 & 106.77 & -17.3297 \\
\hline 7.5 & 39 & 5.0 & 130 & 132 & -29.9242 & 110.2 & -19.1351 \\
\hline 7.5 & 38 & 5.5 & 66 & 87.1 & -22.5029 & 63.95 & 5.259259 \\
\hline 6.5 & 40 & 5.5 & 232 & 80.05 & -16.3023 & 67.625 & -0.93284 \\
\hline 7.0 & 38 & 5.5 & 134.5 & 73 & 0.684932 & 71.3 & 2.993197 \\
\hline & & & & & & & \\
\hline
\end{tabular}

Fig. 4 shows the Pareto diagram of each parameter factor. In the figure, the ordinate is the six parameter factors, and the abscissa is the standardized Pareto response.
The primary effect order of the self-priming time between each parameter can be clearly obtained from the figure. As shown in the Fig. 4, when the significance level $a=0.05$ 
the critical $t$ value is 2.306 , that is, when the $t$ value of the parameter exceeds 2.306, it indicates that the parameter has a significant response to the self-priming time. Therefore, for the main effect, the outlet inner diameter $A$ far exceeds the critical value, that is, outlet inner diameter $A$ has the most significant response to the self-priming time, and the throat pipe inner diameter $C$ also exceeds the critical value, indicating that the throat pipe inner diameter $C$ also shows a significant response to the self-priming time. However, only the connecting sleeve length $B$ does not respond significantly to the self-priming time. Moreover, comparing the interaction between the parameters, it can be found that only the interaction between outlet inner diameter $A$ and the connecting sleeve length $B$ has a significant response to the self-priming time, and its t value is slightly lower than that of the throat pipe inner diameter $C$. What's more, the interaction between the connecting sleeve length $B$ and throat pipe inner diameter $C$ is close to the response of the connecting sleeve length $B$ on the self-priming time. Only the interaction between outlet inner diameter $A$ and throat pipe inner diameter $C$ has the weakest response to the self-priming time. Therefore, the order of response to self-priming time is as follows: outlet inner diameter $A>$ throat pipe inner diameter $C>$ interaction between outlet inner diameter $A$ and the connecting sleeve length $B>$ interaction between the connecting sleeve length $B$ and throat pipe inner diameter $C>$ connecting sleeve length $B>$ interaction between the connecting sleeve length $B$ and throat pipe inner diameter $C>$ interaction between outlet inner diameter $A$ and throat pipe inner diameter $C$. This analysis result is consistent with the response order of the main effects and interactions to the self-priming time described above, and further distinguishes the significance of the self-priming time response.

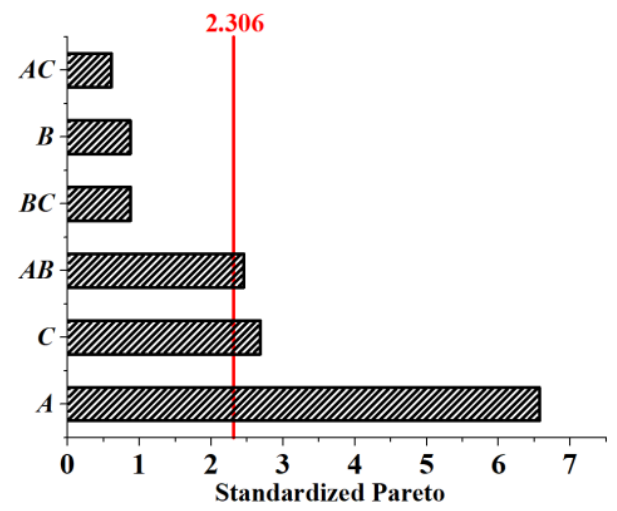

Fig. 4 Standardized Pareto diagram

Considering that only the first-stage nozzle diffuser outlet inner diameter $A$, the second throat pipe inner diameter of second-stage nozzle $C$ and interaction between outlet inner diameter $A$ and the connecting sleeve length $B$ have a significant response to the self-priming time. Therefore, the self-priming performance model can be simplified by deleting the insignificant response parameters interaction between the connecting sleeve length $B$ and throat pipe inner diameter $C$, interaction between outlet inner diameter $A$ and throat pipe inner diameter $C$, and the connecting sleeve length $B$, Therefore, the simplified self-priming performance model is shown as follow:

$$
T_{s 2}=824-97.4 * A-28.2 * C+0.49 A * B .
$$

In order to verify the accuracy of the model, the geometric parameters of outlet inner diameter $A$, connecting sleeve length $B$ and throat pipe inner diameter $C$ were added into the different self-priming performance models to predicate the self-priming time, and the results error between the self-priming test and the model prediction were obtained. It found that the analysis error of self-priming model employing all parameters is obviously larger than the simplified self-priming model. As shown in Table 2, the maximum error before model simplification is $39.3382 \%$, and the simplified error is reduced to $29.93898 \%$, which greatly improves the accuracy of prediction. Due to that the water in the pump cavity needs to be exhausted before the self-priming test, which ensure no water in the pump and every test have the same start test condition. Subsequently, the self-priming nozzle is replaced, considering the entire process is performed under a 5-meter self-priming platform, which lead to the process is very complicated. Therefore, by proposing the self-priming model, the self-priming time of different geometric parameters self-priming nozzles can be effectively predicted, the test analysis cycle and production costs were greatly reduced, which can provide the design reference of self-priming nozzles optimization.

\section{Test analysis of self-priming performance at different heights}

According to the Chinese test standard JB/T 72562013, the maximum flow rate of self-priming pump is 200 $\mathrm{m} 3 / \mathrm{h}$, and the self-priming time is $200 \mathrm{~s}$ when the self-priming height is $5 \mathrm{~m}$. In this paper, flow rate of self-priming pump can reach $500 \mathrm{~m}^{3} / \mathrm{h}$, the self-priming time is only 62.5 $\mathrm{s}$ at self-priming height of $5 \mathrm{~m}$, which can start without water in the pump cavity. In order to analyze the self-priming performance under different self-priming heights, test bench with $5 \mathrm{~m}, 6 \mathrm{~m}, 7 \mathrm{~m}$ and $8 \mathrm{~m}$ was built. Table 3 presents the results of self-priming test.

Table 3

Test of self-priming performance under different height

\begin{tabular}{|l|l|l|l|l|}
\hline Self-priming height, $\mathrm{m}$ & 5 & 6 & 7 & 8 \\
\hline Self-priming time, $\mathrm{s}$ & 62.5 & 92 & 121 & 173 \\
\hline
\end{tabular}

As shown in the Fig. 5, the variation curve of the self-priming time with self-priming height is obtained. Meanwhile, four different self-priming heights were selected as nodes, which generate four smooth transition curves. In the figure, $R$ represents the growth rate of selfpriming time with self-priming height in different selfpriming height ranges. Through comparison, it found that with the continuous increase of self-priming height, the overall self-priming time shows an upward trend. Since the self-priming time shows different growth rate with the variation of the self-priming height, the self-priming process within $8 \mathrm{~m}$ was divided into three stages. The first stage is when the self-priming height is less than $5 \mathrm{~m}$, the self-priming time shows a non-linear growth trend with the increase of the self-priming height, the increase $R_{1}$ is small while the self-priming height increases. In the second stage, when the self-priming height increases to the range of $5 \mathrm{~m}$ to $6 \mathrm{~m}$, the growth rate $R_{2}$ increases significantly as the self-priming height increases. However, in the third stage, when the selfpriming height increases to the range of $6 \mathrm{~m}$ to $8 \mathrm{~m}$, the self- 
priming time presents a linear upward trend with the increase of the self-priming height, and the growth rates $R_{3}$ and $R_{4}$ both remain constant.

The main reason for this phenomenon is that the negative pressure was generated at the inlet of the pump, which causes the air dissolved in the water to continuously precipitate, increasing the air volume of the inlet pipeline. Therefore, in the first and second stages of the start-up process, the self-priming time exhibits different degrees of non-linear growth with the increase of the self-priming height. With the continuous increase of the self-priming height, the air in the water of the inlet pipe starts to precipitate, which makes the corresponding air volume continue to decrease. However, when the self-priming height larger than $6 \mathrm{~m}$, the volume of air in the inlet pipe released during the self-priming process is very small, and the total amount of air in the inlet pipe is constant. Therefore, in the range of the self-priming height from $6 \mathrm{~m}$ to $8 \mathrm{~m}$, the self-priming time shows a linear growth relationship with the increase of the self-priming height.

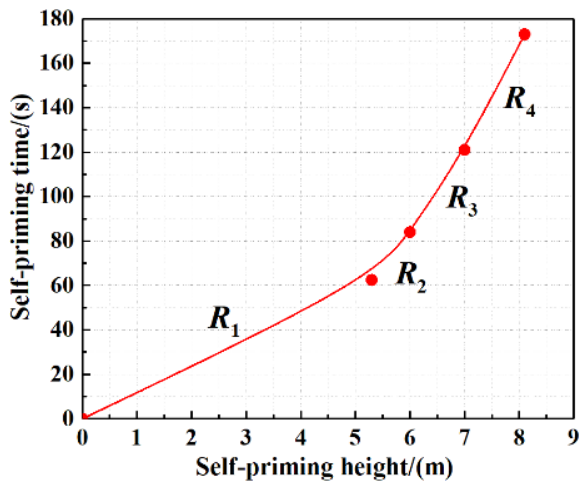

Fig. 5 The relationship between self-priming time and altitude

\section{Conclusions}

In this paper, experiments were conducted to optimize the self-priming performance of self-priming nozzle, and the full factor test method is employed to explore the main and interaction effect of the nozzle geometric parameters on the self-priming performance, and the conclusions can be obtained as follow:

1. In this paper, the full factor test method is employed to explore the main and interaction effect of the nozzle geometric parameters on the self-priming performance. This method can not only analyze the main effects of the parameters, but also effectively evaluate the interaction between the parameters. Therefore, according to the analysis results of full factor test, the order of response to self-priming time is obtained: outlet inner diameter $A>$ throat pipe inner diameter $C>$ interaction between outlet inner diameter $A$ and the connecting sleeve length $B>$ interaction between the connecting sleeve length $B$ and throat pipe inner diameter $C>$ connecting sleeve length $B>$ interaction between the connecting sleeve length $B$ and throat pipe inner diameter $C>$ interaction between outlet inner diameter $A$ and throat pipe inner diameter $C$.

2. Based on the analysis results of the full factor test, a novel self-priming performance model with outlet inner diameter $A$, connecting sleeve length $B$ and throat pipe inner diameter $C$ as variables was established. By simplifying the self-priming performance model, the maximum error is reduced from $39.3382 \%$ to $29.93898 \%$, which greatly improves the accuracy of prediction. Therefore, the self-priming time of different geometric parameters self-priming nozzles can be effectively predicted, the test analysis cycle and production costs were greatly reduced, which can provide the design reference of self-priming nozzles optimization.

3. The self-priming performance test with different self-priming heights were conducted, the variation regulation between self-priming time and self-priming height was obtained, it found that with the continuous increase of selfpriming height, the overall self-priming time shows an upward trend. Considering the negative pressure was generated at the inlet of the pump, which causes the air dissolved in the water to continuously precipitate, increasing the air volume of the inlet pipeline. Therefore, the self-priming time shows three different growth rates with the variation of the self-priming height.

\section{Acknowledgements}

The authors gratefully acknowledge the support from Open Research Fund Program of State key Laboratory of Hydroscience and Engineering grant number: sklhse2020-E-01; Open Research Subject of Key Laboratory of Fluid Machinery and Engineering (Xihua University) grant number LTJX2021-003; Open Research Subject of Hubei Key Laboratory of Hydroelectric Machinery Design \& Maintenance (China Three Gorges University) grant number 2020KJX07; The 69th batch of general funding from the China Postdoctoral Science Foundation grant number: 2021M691298; Priority Academic Program Development of Jiangsu Higher Education Institutions (PAPD).

\section{References}

1. Chang, H.; Agarwal, R. K.; Li, W. et al. 2019. Numerical and experimental study of a vortex structure and energy loss in a novel self-priming pump, Processes 7(10): 701.

2. Wu, D.; Zhu, Z.; Ren, Y. et al. 2019. Influence of blade profile on energy loss of sewage self-priming pump, Journal of the Brazilian Society of Mechanical Sciences and Engineering 41(10): 470.

3. Zhou, P.; Wu, Z.; Mou, J. et al. 2019. Effect of reflux hole on the transient flow characteristics of the selfpriming sewage centrifugal pump, Journal of Applied Fluid Mechanics, 12(3): 689-699.

4. Yao, H. Y.; Zhang, Y. D.; Wu, D. Z. et al. 2018. Numerical study on hydraulic and self-priming performance of a double-stage self-priming pump, IOP Conference Series: Earth and Environmental Science, IOP Publishing 163(1): 012039.

5. Zhang, Y. L.; Zhu, Z. C.; Zhao, Y. J. et al. 2020. Comparative experiments on a self-priming pump delivering water medium during rapid and slow starting periods, Iranian Journal of Science and Technology, Transactions of Mechanical Engineering 1-13.

6. Wang, C.; He, X.; Zhang, D. et al. 2019. Numerical and experimental study of the self-priming process of a multistage self-priming centrifugal pump, International Journal of Energy Research 43(9): 4074-4092.

7. Chang, H.; Shi, W.; Li, W. et al. 2019. Experimental optimization of jet self-priming centrifugal pump based 
on orthogonal design and grey-correlational method, Journal of Thermal Science, p. 1-10.

H. Chang, Sh. Hong, D. Yu, G. Peng, D. Ye, J. Liu, Sh. Yuan

EXPERIMENTAL OPTIMIZATION OF SELFPRIMING PUMP BASED ON THE FULL FACTOR TEST

S u m m a r y

Self-priming pump as the core equipment for flood fighting and emergency rescue plays an important role in flood prevention and other fields. More importantly, the geometric parameters of the self-priming nozzle directly determine the performance of the self-priming pump. In the previous analysis, only small amount of experiment was conducted to optimize the geometry parameters of selfpriming nozzle, and the interaction between the parameters was not considered. In this paper, the full factor test method is employed to explore the main and interaction effect of the nozzle geometric parameters on the self-priming performance, and the order of response to self-priming time is obtained. Meanwhile, a novel self-priming performance model was established, the self-priming time of different geometric parameters self-priming nozzles can be effectively predicted, and the test analysis cycle and production costs were greatly reduced. Finally, the self-priming test under different self-priming height with optimal self-priming nozzle was conducted, the variation regulation between self-priming time and self-priming height was obtained. Therefore, this research could effectively provide the design reference of self-priming nozzles optimization.

Keywords: experimental optimization, self-priming pump, prediction model, full factor test.

Received November 16, 2020

Accepted October 04, 2021

This article is an Open Access article distributed under the terms and conditions of the Creative Commons Attribution 4.0 (CC BY 4.0) License (http://creativecommons.org/licenses/by/4.0/). 